\title{
Un ensayo clínico aleatorizado de farmacoterapia con monitorización telefónica para mejorar el tratamiento de la depresión en la atención primaria en Santiago, Chile
}

\author{
Rosemarie Fritsch ${ }^{1}$, Ricardo Araya ${ }^{3}$, Jaime Solís ${ }^{1}$, \\ Elena Montt ${ }^{1}$, D aniel Pilowsky² ${ }^{2}, \mathrm{G}$ raciela Rojas ${ }^{1}$. \\ A randomized trial of pharmacotherapy \\ with telephone monitoring to improve \\ treatment of depression in primary care \\ in Santiago, Chile
}

Background: Depression is a public health problem, due to its high prevalence and its associated disability. Aim: To compare a pharmacological intervention for depression controlled by phone from a central level (TM) and the usual treatment (TH) in a randomized clinical trial. Material and methods: Three hundred and forty five women, aged 22 to 59 years were studied. They were randomly assigned to receive the usual therapy or a pharmacological intervention with periodical telephone contacts with medical collaboration personnel, to reinforce compliance with treatment and educate about the disease. Women were blindly evaluated at 3 and 6 months with the Hamilton depression rating score (HDRS) and the SF-36 to assess depressive symptoms and quality of life, respectively. Results: In both evaluations, improvement was significantly greater in the TM group than the TH group. At 3 months, improvement was higher in the TM group in the subscales of physical function, pain, general health, energy, emotional role, mental health and standardized physical and psychic scales of SF36. At 6 months, this significant difference in favour of TM was maintained for energy, mental health and the standardized psychic scale. Conclusions: A telephone reinforcement improves the outcomes of treatments for depression (Rev Méd Chile 2007; 135: 587-95).

(Key words: Compliance; Depression; Drug therapy)

Recibido el 18 de julio, 2006. Aceptado el 24 de octubre, 2006.

Trabajo financiado por Fondecyt, Chile, Proyecto FONDECYT \# 1040432.

Hospital Clínico de la Universidad de Chile, Santiago de Chile. Columbia University, New York, USA, University of Bristol, UK.

Correspondencia a: Dra. Graciela Rojas. Directora Clínica

Psiquiátrica Universitaria, Universidad de Chile, Campus

Norte. Av. La Paz 1003, Santiago, Chile.

E mail:grojas@redmedicauchile.cl 
$\mathrm{L}$ os trastornos psiquiátricos, y en particular el trastorno depresivo, son altamente prevalentes en la población general. Estos últimos, por su alta frecuencia y grado de discapacidad que conllevan, producen altos costos para la sociedad ${ }^{1-5}$. Estudios de la Organización Mundial de la Salud (OMS) señalan que la depresión daba cuenta, en 1990 , de $11 \%$ de los años de vida con discapacidad (YLDs) y constituía la cuarta causa de años de vida ajustados por discapacidad (DALYSs), proyectándose que para el 2020 se transformaría en la segunda causa global y la primera en mujeres ${ }^{3,6}$.

En Chile se han realizado dos estudios de prevalencia de trastornos psiquiátricos en la población general. El estudio de salud mental en el Gran Santiago, que utilizó la entrevista clínica estructurada en su versión revisada -CIS-R- e informó cifras de prevalencia semanal de cualquier trastorno, de acuerdo a la clasificación internacional de las enfermedades -CIE-10- de $23,99 \%$ y de $5,5 \%$ (2,7\% en hombres y $8,0 \%$ en mujeres) para episodio depresivo en la población adulta del Gran Santiago" ${ }^{1}$. El otro fue el "Estudio chileno de prevalencia de patología psiquiátrica", realizado en cuatro provincias de Chile que utilizó los criterios del «iagnostic and Statistical Manual for Mental Disorders. $3^{\text {rd }}$ ed. Revised»-DSM-III Rusando la Composite international diagnostic interview (CIDI) y que informó una prevalencia semestral de patología psiquiátrica de 22,6\%, de trastornos afectivos de 7,9\% y de trastorno depresivo mayor de 4,6\% (3,0 en hombres y 6,0 en mujeres $)^{4,5}$.

Los trastornos depresivos son frecuentes en la atención primaria. Estudios epidemiológicos en Estados Unidos de Norteamérica, Europa Occidental, así como el estudio multicéntrico llevado a cabo por la Organización Mundial de la Salud en 14 países del mundo, han encontrado cifras entre $8 \%$ y $12 \%$ de prevalencia de depresión mayor en pacientes adultos de la atención primaria ${ }^{7}$. Las investigaciones epidemiológicas llevadas a cabo en Chile, han arrojado cifras de prevalencia cercanas a $30 \% 8$.

A pesar de la evidencia acumulada sobre la efectividad de los tratamientos antidepresivos, un porcentaje importante de pacientes no reciben tratamiento adecuado y, si lo hacen, no lo continúan por el tiempo necesario 9,10. La depresión es un cuadro generalmente de varios meses de evolución y que, en algunos pacientes, presenta un curso crónico o recidivante, observándose una recurrencia de 50\% luego de un primer episodio, de 80\% luego de un segundo, y alrededor de 90\% luego de un tercer episidio $^{10-12}$. Los problemas para evitar las recaídas o recurrencias han demostrado ser importantes ${ }^{10,13}$.

En los últimos 15 años, el Ministerio de Salud chileno ha implementado dos Planes Nacionales de Salud Mental y Psiquiatría ${ }^{14,15}$.

El Plan Nacional de Salud Mental y Psiquiatría del año 2000 consideró un Programa Nacional para la Detección, Diagnóstico y Tratamiento de la Depresión (PNDDTD) como un reconocimiento a la creciente importancia del fenómeno de la depresión como uno de los principales problemas de salud pública de nuestro país. Este programa, cuya aplicación comenzó el año 2000 en la atención primaria, buscó dar respuestas a este problema de salud en el lugar a donde concurre el $75 \%$ de las personas que sufren depresión ${ }^{15,16}$.

El programa nacional para la detección, diagnóstico y tratamiento integral de la depresión ${ }^{16}$ consiste en un conjunto de sugerencias de atención que combina intervenciones médicas y psicosociales, con actividades que incluyen la detección, el diagnóstico, el registro, el tratamiento y el control de cada caso. Estudios internacionales indican, sin embargo, que la simple implementación de guías clínicas ha resultado ser inefectiva para el tratamiento de la depresión ${ }^{17}$. Las estrategias basadas en la implementación de guías clínicas destinadas a mejorar el diagnóstico y tratamiento de la depresión, sólo resultan efectivas si se acompañan de intervenciones organizacionales más complejas como manejo de casos, cuidados colaborativos, programas estructurados e iniciativas para mejorar la calidad del servicio ${ }^{18-21}$.

La presente comunicación informa los resultados de un ensayo clínico que comparó aleatoriamente un tratamiento a mujeres deprimidas con farmacoterapia estructurada y monitorizada telefónicamente, con el tratamiento habitual que se lleva a cabo en la atención primaria de acuerdo a los lineamientos ministeriales.

\section{Metodología}

Se llevó a cabo un ensayo clínico aleatorio en 5 consultorios de la atención primaria de Santiago. 
Se comparó una intervención de farmacoterapia monitorizada (FM) con el modelo de tratamiento actualmente en uso (TH) en la atención primaria (AP) (Figura 1).

La intervención de la farmacoterapia monitorizada comprendió visitas periódicas al médico general con farmacoterapia estructurada mediante fichas clínicas con algoritmos y contactos telefónicos periódicos por parte de personal no profesional entrenado, con el fin de realizar educación, monitorizar el consumo de psicofármacos y efectos colaterales y reforzar la asistencia a controles médicos. Se utilizaron los antidepresivos que están disponibles en la atención primaria (fluoxetina, amitriptilina, imipramina). El personal encargado de la monitorización recibió un entrenamiento de 2 $\mathrm{h}$, los médicos generales de $6 \mathrm{~h}$ antes del inicio del estudio y ambos recibieron monitorización permanente por parte del equipo investigador. Los médicos asistieron a reuniones mensuales con un psiquiatra, para discusión de casos.

El tratamiento habitual se basó en el programa del Ministerio de Salud para el tratamiento de la depresión en la atención primaria, que incluye controles con médicos generales, farmacoterapia,

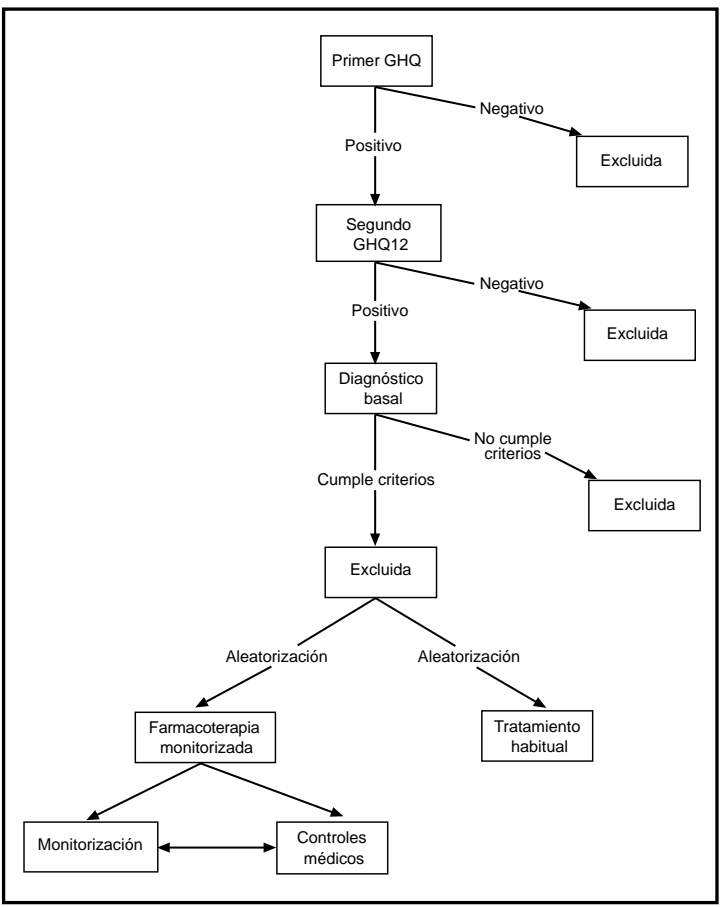

Figura 1. Diseño del estudio. psicoterapia individual o grupal con psicólogos e interconsultas con psiquiatras ${ }^{16}$.

Reclutamiento. Se realizó un proceso de detección de mujeres adultas (entre 18 y 70 años) consultantes a los consultorios con episodio de depresión mayor en curso, sin tratamiento en los últimos 3 meses y con, al menos, 1 hijo entre 6 y 16 años viviendo con ella. Este criterio de selección se debe a que se realizó un seguimiento de los hijos de estas mujeres, con el objetivo de evaluar el impacto de la mejoría sobre la salud mental de los niños. Se excluyeron a las mujeres que tuviesen: abuso de alcohol o drogas, antecedente de enfermedad bipolar, sintomatología psicótica actual o en el pasado, condición discapacitante mental o física que le impidiera participar en las actividades y evaluaciones del estudio, embarazo y alto riesgo de suicidio.

El procedimiento de tamizaje comenzó en la sala de espera de los consultorios utilizando el Cuestionario de Salud de Goldberg (GHQ-12). Las mujeres que obtenían un puntaje mayor a 4 eran invitadas al consultorio para una segunda evaluación en dos semanas. Aquellas que persistían con un puntaje mayor a 4 puntos, eran invitadas a participar en el diagnóstico basal, previa firma de un consentimiento informado. El GHQ-12 es un instrumento breve para medir malestar psíquico y que ha sido validado y usado en nuestro país en investigaciones anteriores llevadas a cabo en la atención primaria, describiéndose una sensibilidad de $78 \%$ y un valor predictivo de $55 \% 22,23$.

Aleatorización. Las pacientes fueron asignadas en forma aleatoria y ciega a una de las dos modalidades de tratamiento. Esta se llevó a cabo a nivel individual, usando sistemas computacionales administrados desde un nivel central.

Las mujeres fueron evaluadas basalmente con el Mini International Neuropsychiatric Interview (MINI), una entrevista diagnóstica estructurada, de corta duración que explora de manera estandarizada cada uno de los criterios necesarios para el establecimiento de los diagnósticos principales de la Clasificación Internacional de las Enfermedades (Organización Mundial de la Salud, 1993). Puede ser utilizado por clínicos, especialistas o no, que hayan recibido una formación previa ${ }^{24}$. Se aplicó la sección para el diagnóstico de depresión mayor, preguntas de tamiza- 
je, extraídas del MINI, sobre síntomas psicóticos y maníacos presentes actualmente y en el pasado, secciones del MINI para detectar dependencia al alcohol y drogas, riesgo de suicidio, un conjunto de preguntas de variables sociodemográficas, incluido el censo de los hijos, una entrevista para obtención de antecedentes familiares de patología psiquiátrica en general, y trastornos afectivos en particular, la Escala de Depresión de Hamilton (HRSD) ${ }^{25}$. Estudios en la atención primaria indican que este instrumento presenta una buena confiabilidad y validez convergente en comparación con otras escalas de depresión y el Cuestionario de Calidad de Vida (SF36) que contiene 36 ítems, formando 8 dimensiones que evalúan función física, rol físico, dolor corporal, salud general, vitalidad, función social, rol emocional y salud men$\mathrm{tal}^{26}$. Es un buen instrumento para medir calidad de vida y ha sido utilizado en estudios previos ${ }^{27}$.

Evaluaciones de seguimientos. Las pacientes de ambos grupos fueron evaluadas a los 3 y 6 meses en forma ciega con el HDRS, el SF-36 y un cuestionario de usos de servicios.

Estadística. El análisis estuvo en concordancia con las guías CONSORT sobre la base de "intención de tratar". Se llevaron a cabo: a) estadísticas descriptivas de las variables sociodemográficas y clínicas de las mediciones basales para detectar cualquier diferencia entre los grupos, b) análisis de regresiones múltiples usando la variable principal -HDRS- como una variable continua, y controlando por los valores basales, la edad y el consultorio. La significación estadística se fijó en 0,05. Todos los cálculos se realizaron con el programa Stata $8.0^{28}$.

\section{Resultados}

Se realizó la evaluación inicial a 2.358 madres consultantes en forma consecutiva, resultando positivo en $57,9 \%$ de los casos. El $20,7 \%$ de la muestra original obtuvo un segundo puntaje positivo en el GHQ12. La mayoría de ellas completó la evaluación basal. El 27,1\% fue excluido por diversas razones. Sólo un pequeño porcentaje (2,3\%) rechazó participar en el estudio. El promedio del puntaje del GHQ12 de aquellas 821 que no volvieron a la segunda evaluación fue de 8,3 (D.E. 2,3), mientras que el promedio de las que realizaron el segundo GHQ12 fue de 8,7 (D.E. 3,8). Se observa una diferencia estadísticamente significativa, lo que sugiere que aquellas mujeres que no regresaron tuvieron una remisión espontánea de los síntomas.

Finalmente, 345 mujeres aceptaron participar en el estudio, las que fueron aleatorizadas a las dos formas de tratamiento (Figura 2).

Descripción de la muestra. La muestra estuvo constituida por 345 mujeres con edad promedio de 37,4 años (CI 95\%: 36,6-38,1). El rango etario fue amplio y la distribución no fue normal. La mediana de la edad fue 37 años y el rango de 22 a 59 años. Aproximadamente la mitad de ellas estaban casadas, un quinto convivía y otro quinto era separada o soltera. La gran mayoría era dueña de casa y un tercio trabajaba. El 25,4\% de las dueñas de casa, además, trabajaba. Algo más de la mitad de las mujeres refería haber tenido, al menos, un episodio previo de depresión. El puntaje promedio en la

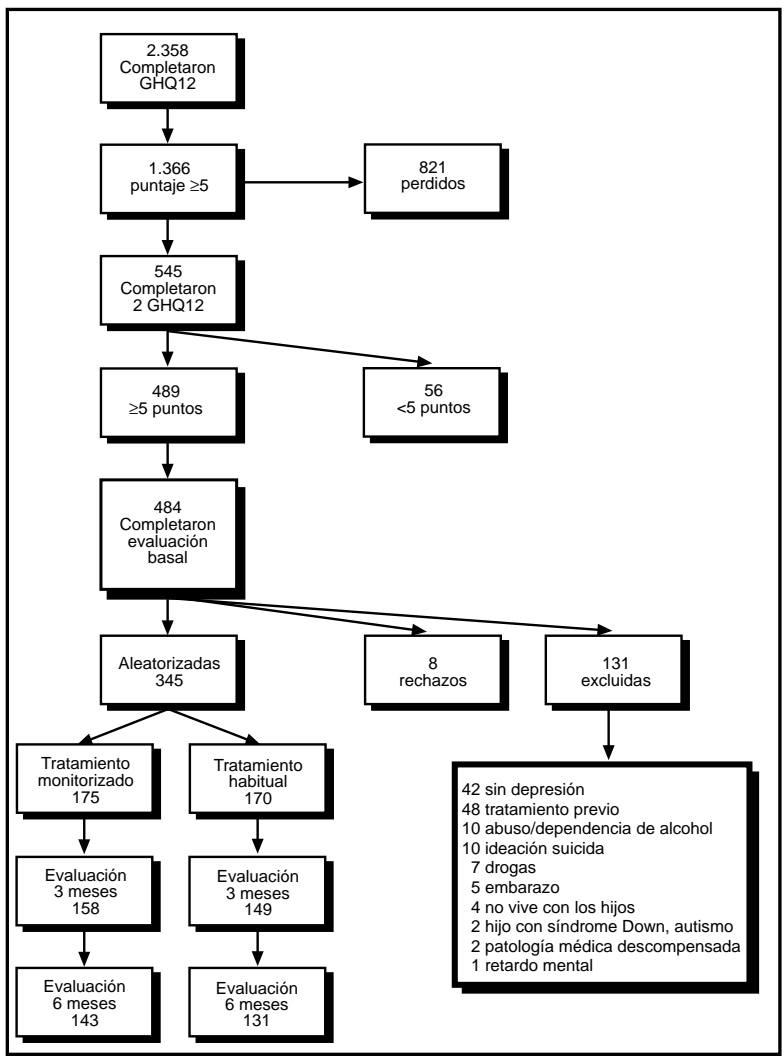

Figura 2. Flujo del estudio. 
escala de Hamilton fue de 19,1 (18,6-19,7). Ambos grupos no se diferenciaron significativamente en sus variables sociodemográficas, antecedentes clínicos y calidad de vida (Tabla 1).

Efectividad de la respuesta antidepresiva. A los 3 meses de tratamiento $81,0 \%$ de las pacientes de la FM y $30,2 \%$ del TH había tomado antidepresivos $\left(\mathrm{chi}^{2}=80,492 ; \mathrm{p}=0,000\right)$. A los 6 meses de tratamiento $71,6 \%$ de las en FM y $25,2 \%$ del TH había recibido fármacos en el último período ( $\mathrm{chi}^{2}=58,6 ; \mathrm{p}=0,000$ ). El $84,2 \%$ de las pacientes de la FM y 36,9\% del TH recibió en algún momento fármacos antidepresivos $\left(\mathrm{chi}^{2}=72,2 ; \mathrm{p}=0,000\right)$.

Ambos grupos presentaron una mejoría significativa tanto a los 3 como a los 6 meses de

Tabla 1. Características basales

\begin{tabular}{|c|c|c|c|c|}
\hline Características & $\begin{array}{l}\text { Muestra Total } \\
\qquad \mathrm{N}=345\end{array}$ & $\begin{array}{c}\mathrm{FM} \\
\mathrm{N}=175 \\
\end{array}$ & $\begin{array}{c}\mathrm{TH} \\
\mathrm{N}=170 \\
\end{array}$ & $\mathrm{p}$ \\
\hline Edad (años) promedio (95\% IC) & $37,4(36,6-38,1)$ & $3,5(36,4-38,5)$ & $37,2(36,2-38,3)$ & 0,7742 \\
\hline \multicolumn{5}{|l|}{ Estado civil (\%) (95\% IC) } \\
\hline solteras & $9,3(6,3-13,1)$ & $0,1(0,05-0,15)$ & $9,4(5,4-15,3)$ & \\
\hline casadas & $54,8(47,2-63,2)$ & $55,1(44,4-67,6)$ & $54,7(44,1-67,0)$ & \\
\hline convivientes & $21,4(16,8-26,9)$ & $19,8(13,6-27,7)$ & $22,9(16,3-31,4)$ & 0,9000 \\
\hline separadas & $13,3(9,8-17,8)$ & $15,0(9,7-22,1)$ & $11,8(7,2-18,2)$ & \\
\hline viudas & $1,2(0,3-3,0)$ & $1,2(0,1-4,3)$ & $1,2(0,1-4,2)$ & \\
\hline \multicolumn{5}{|l|}{ Ocupación (\%) (95\% IC) } \\
\hline $\begin{array}{l}\text { dueña } \\
\text { de casa }\end{array}$ & $90,1(86,5-93,1)$ & $91,6(86,3-95,3)$ & $90,0(84,5-94,1)$ & 0,7071 \\
\hline estuc & $3,8(2,0-6,4)$ & $5,4(2,5-10,0)$ & $2,3(0,6-5,9)$ & 0,1680 \\
\hline traba & $30,4(25,6$ & 29,3 & $31,2(24$, & 0,7237 \\
\hline cesant & $6,7(4,3-9,8)$ & $7,8(4,2-12,9)$ & $5,9(2,9-10,5)$ & 0,5237 \\
\hline $\begin{array}{l}\text { Número de hijos } \\
\text { promedio ( } 95 \% \text { IC) }\end{array}$ & $2,7(2,5-2,8)$ & $2,7(2,5-2,9)$ & $2,6(2,5-2,8)$ & 0,7483 \\
\hline HDRS & & & & \\
\hline $\begin{array}{l}\text { promedio }(95 \% \text { lC) } \\
\text { SF-36 función física }\end{array}$ & $19,1(18,6$ & ,) & t) & \\
\hline $\begin{array}{l}\text { promedio ( } 95 \% \text { IC) } \\
\text { SF-36 rol físico }\end{array}$ & $69,7(67,2-72,3)$ & $69,9(66,3-73,5)$ & $69,5(65,9-73,2)$ & 0,8938 \\
\hline promedio (95\% IC) & $41,5(37,5-45,6)$ & $42,4(36,5-48,3)$ & $40,7(35,1-46,3)$ & 0,6794 \\
\hline $\begin{array}{l}\text { SF-36 dolor } \\
\text { promedio (95\% IC) }\end{array}$ & $39,4(36,7-42,0)$ & $39,0(35,4-42,6)$ & $39,7(35,7-43,7)$ & 0,7953 \\
\hline $\begin{array}{l}\text { SF-36 salud general } \\
\text { promedio (95\% IC) }\end{array}$ & $43,0(41,2-44,8)$ & $43,5(40,9-46,0)$ & $42,5(39,8-45,2)$ & 0,6049 \\
\hline SF-36 vitalidad & & & & \\
\hline promedio (95\% IC) & $33,2(31,6-34,8)$ & $32,4(30,2-34,5)$ & $34,1(31,7-36,5)$ & 0,2887 \\
\hline $\begin{array}{l}\text { SF-36 funcionamiento social } \\
\text { promedio ( } 95 \% \text { IC) }\end{array}$ & $46,5(43,5-49,4)$ & $46,2(42,1-50,3)$ & $46,7(42,5-51,0)$ & 0,8537 \\
\hline $\begin{array}{l}\text { SF-36 rol emocional } \\
\text { promedio ( } 95 \% \text { IC) }\end{array}$ & & & & \\
\hline SF-36 salud mental & 25 & $2 J, j\left(20, T^{-} J_{1} T\right.$ & 1) & \\
\hline promedio (95\% IC) & $34,8(33,3-36,3)$ & $33,9(31,9-36,0)$ & $35,7(33,5-37,8)$ & 0,2499 \\
\hline $\begin{array}{l}\text { SF-36 escala estandarizada } \\
\text { física ( } 95 \% \text { IC) }\end{array}$ & $42,6(41,6-43,7)$ & $42,7(41,3-44,1)$ & $42,6(41,1-44,1)$ & 0,8870 \\
\hline $\begin{array}{l}\text { SF-36 escala estandarizada } \\
\text { psíquica ( } 95 \% \text { IC) } \\
\text { antecedentes de depresión }\end{array}$ & $28,7(27,8-29.6)$ & $28,6(27,3-30,0)$ & $28,8(27,5-30,1)$ & 0,8878 \\
\hline$\%(95 \%$ IC) & $55,6(50,2-61,0)$ & $57,8(49,9-65,4)$ & $54,5(46,6-62,2)$ & 0,5813 \\
\hline
\end{tabular}


tratamiento. Sin embargo, en ambos momentos de evaluación, el grado de mejoría fue significativamente mayor en el grupo TM respecto del TH, incluso controlando por el puntaje inicial de Hamilton, el consultorio y edad de la paciente en un modelo de regresión múltiple (Tabla 2). Al incluir en el modelo la utilización de fármacos, no se observó una correlación significativa con el puntaje en la escala de Hamilton, al controlar por tipo de tratamiento, consultorio y edad de las pacientes.

Tratamiento antidepresivo y calidad de vida. A los 3 meses de tratamiento se observó una mejoría mayor en el grupo con TM en varias subescalas del SF-36. Estos son función física, dolor, salud general, vitalidad, rol emocional, salud mental y escalas estandarizadas física y psíquica. A los 6 meses de tratamiento esta diferencia significativa a favor del TM se mantuvo para la vitalidad, salud mental y escala estandarizada psíquica (Tabla 3).

\section{DisCUSIÓN}

Se trata de un ensayo clínico randomizado que entrega evidencias sobre posibles medidas que se pueden llevar a cabo en el manejo de la depresión en la atención primaria y que mejoraría el tratamiento de una enfermedad importante para la salud pública.

En la atención primaria chilena se demostró que en mujeres, un tratamiento escalonado y estructurado en que las personas con depresión leve eran tratadas con una intervención grupal de orientación cognitiva conductual y a las personas con depresiones más graves se les administraba además fluoxetina, era factible y eficaz en la disminución de la sintomatología depresiva y en la mejoría del funcionamiento de las mujeres tratadas con esta modalidad ${ }^{27}$. Este ensayo clínico no podía determinar la contribución de los distintos componentes del programa a la efectividad de los resultados. Los resultados del presente estudio permitirían simplificar las intervenciones que se llevan a cabo en los consultorios para enfrentar la enfermedad depresiva.

Las intervenciones más efectivas, según estudios internacionales, son complejas e incorporan educación, manejo de casos con monitorización por parte de personal de enfermería, consejería y monitorización telefónica de la farmacoterapia y una integración entre el nivel primario y secundario ${ }^{18-21,29,30}$.

El manejo de otras enfermedades crónicas ha mostrado que intervenciones simples como sistemas de ayuda memoria y seguimiento telefónico aumentan la adherencia de los médicos a las guías clínicas, la adherencia de las personas a su tratamiento, mejoran el manejo de las enfermedades crónicas y apoyan cambios en la conducta de salud de las personas ${ }^{18,19,31-33}$. En el caso de la enfermedad depresiva, se han utilizado, a nivel internacional, diversas estrategias para optimizar el tratamiento en atención primaria: uso de guías de tratamiento, manejo y seguimiento de cada caso por personal no médico, interconsultas con psiquiatra, sistemas de recuerdo automático, información entregada junto a los fármacos, educación a los pacientes o médicos primarios, etc. Los resultados de distintos estudios controlados randomizados utilizando uno o varios de estos componentes, han concluido que una de las intervenciones que incide de mayor manera en la adherencia y el pronóstico es alguna forma de seguimiento estructurado. En varios estudios este seguimiento fue realizado a un bajo costo, vía telefónica, por enfermera o personal auxiliar de enfermería. Simon y colaboradores han descrito el seguimiento estructurado ${ }^{20,21}$ que asegura que los pacientes son manejados en forma activa y sistemática de modo tal que se mantiene

Tabla 2. Respuesta antidepresiva (H D RS). Puntajes de seguimiento según randomización*

\begin{tabular}{|crrc|}
\hline & \multicolumn{1}{c}{ FM } & \multicolumn{1}{c|}{ TH } & $\mathrm{p}$ \\
\hline 3 meses & $9,4(8,4-10,4)$ & $12,4(11,2-13,5)$ & 0,000 \\
6 meses & $12,8(11,5-14,0)$ & $14,8(13,4-16,2)$ & 0,015 \\
\hline
\end{tabular}

*Regresión lineal jerárquica con efectos aleatorios: controlando por HDRS basal, consultorios y edad, datos observados. 
Tabla 3. C alidad de vida (SF-36). Puntajes de seguimiento según aleatorización*

\begin{tabular}{|c|c|c|c|}
\hline & FM & $\mathrm{TH}$ & $\mathrm{p}$ \\
\hline \multicolumn{4}{|l|}{$\begin{array}{l}\text { SF-36 función física } \\
\text { promedio (95\% IC) }\end{array}$} \\
\hline 3 meses & $88,1(85,2-91,1)$ & $82,3(78,9-85,6)$ & 0,0181 \\
\hline 6 meses & $80,9(77,7-84,1)$ & $82,3(78,7-85,9)$ & 0,7109 \\
\hline \multicolumn{4}{|l|}{ SF-36 rol físico } \\
\hline \multicolumn{4}{|l|}{ promedio (95\% IC) } \\
\hline 3 meses & $79,3(73,8-84,7)$ & $70,3(63,9-76,7)$ & 0,0534 \\
\hline 6 meses & $53,8(46,8-60,9)$ & $48,7(41,1-56,2)$ & 0,3049 \\
\hline \multicolumn{4}{|l|}{ SF-36 dolor } \\
\hline 3 meses & $73,4(69,9-76,9)$ & $67,0(63,5-70,4)$ & 0,0169 \\
\hline 6 meses & $53,1(49,1-57,2)$ & $50,7(45,9-55,4)$ & 0,3062 \\
\hline \multicolumn{4}{|l|}{$\begin{array}{l}\text { SF-36 salud general } \\
\text { promedio (95\% IC) }\end{array}$} \\
\hline 3 meses & $65,7(61,9-69,5)$ & $56,5(52,6-60,5)$ & 0,0038 \\
\hline 6 meses & $54,7(51,3-58,1)$ & $51,0(47,0-55,0)$ & 0,2143 \\
\hline \multicolumn{4}{|l|}{ S F-36 vitalidad } \\
\hline \multicolumn{4}{|l|}{ Promedio (95\% IC) } \\
\hline 3 meses & $52,1(48,7-55,5)$ & $44,4(41,0-47,8)$ & 0,0017 \\
\hline 6 meses & $48,9(45,4-52,5)$ & $42,3(38,7-45,9)$ & 0,0083 \\
\hline \multicolumn{4}{|c|}{$\begin{array}{l}\text { SF-36 funcionamiento social } \\
\text { promedio ( } 95 \% \text { IC) }\end{array}$} \\
\hline 3 meses & $84,3(80,3-88,4)$ & $77,9(73,3-82,5)$ & 0,0738 \\
\hline 6 meses & $69,2(65,4-73,1)$ & $63,8(59,5-68,2)$ & 0,0750 \\
\hline \multicolumn{4}{|l|}{$\begin{array}{l}\text { SF-36 rol emocional } \\
\text { promedio (95\% IC) }\end{array}$} \\
\hline 3 meses & $70,0(63,5-76,5)$ & $56,4(49,4-63,4)$ & 0,0147 \\
\hline 6 meses & $41,9(34,8-49,0)$ & $34,1(27,0-41,2)$ & 0,1463 \\
\hline \multicolumn{4}{|l|}{$\begin{array}{l}\text { SF-36 salud mental } \\
\text { promedio }(95 \% \text { IC) }\end{array}$} \\
\hline 3 meses & $61,9(59,0-64,8)$ & $54,0(51,1-57,0)$ & 0,0001 \\
\hline 6 meses & $52,6(49,2-56,1)$ & $46,9(43,6-50,2)$ & 0,0091 \\
\hline \multicolumn{4}{|c|}{$\begin{array}{l}\text { SF-36 escala estandarizada física } \\
\text { (95\% IC) }\end{array}$} \\
\hline 3 meses & $51,5(50,2-52,8)$ & $49,1(47,7-50,6)$ & 0,0376 \\
\hline 6 meses & $46,4(44,9-48,0)$ & $46,4(44,6-48,1)$ & 0,9049 \\
\hline \multicolumn{4}{|c|}{$\begin{array}{l}\text { SF-36 escala estandarizada psíquica } \\
\text { (95\% IC) }\end{array}$} \\
\hline 3 meses & $44,0(42,2-45,7)$ & $39,6(37,8-41,5)$ & 0,0008 \\
\hline 6 meses & $38,2(36,2-40,1)$ & $34,2(32,3-36,2)$ & 0,0057 \\
\hline
\end{tabular}

*Regresión lineal jerárquica con efectos aleatorios: controlando por puntaje basal, consultorios y edad, datos observados.

un contacto entre los pacientes y el servicio de salud que los atiende hasta su recuperación. Este seguimiento estructurado tiene elementos educativos y controles descritos por Weingarten y colaboradores $^{34}$ y puede ser llevado a cabo por personal no universitario. La enfermedad depresiva se caracteriza por una falta de motivación y una baja autoestima, lo que pudiese inhibir la asistencia al consultorio. Los médicos de atención primaria tienden a dejar a los pacientes y al personal 
administrativo la decisión de uso de servicios. Si los pacientes no acuden a control con la periodicidad requerida, no hay forma de alertar al médico de esta situación. Una de las razones por la cual el seguimiento estructurado es efectivo es porque aumenta la adherencia al tratamiento farmacológico.

Alvarado y colaboradores, en una evaluación que llevó a cabo del "Programa para la Detección, Diagnóstico y Tratamiento Integral de la Depresión en atención primaria" chilena, proponen simplificar algunas intervenciones que se llevan a cabo e introducir mecanismos de monitorización ${ }^{35}$. Los resultados de este ensayo clínico entregan eviden-

\section{REFERENCIAS}

1. Araya R, Rojas G, Fritsch R, Acuña J, Lewis G. Common mental disorders in Santiago, Chile: prevalence and socio-demographic correlates. $\mathrm{Br}$ J Psychiatry 2001; 178: 228-33.

2. Jenkins R, Lewis G, Bebbington P, Brugha T, Farrell M, Gill B et Al. The National Psychiatric Morbidity surveys of Great Britain: initial findings from the household survey. Psychol Med 1997; 27: 775-90.

3. Murray C, López A. Alternative projections of mortality and disability by cause 1900-2020: Global Burden of Disease Study. Lancet 1997; 349: 1498-504.

4. Vicente B, Saldivia S, Rioseco P, Vielma M, Escobar B, Medina E et al. Trastornos psiquiátricos en diez comunas de Santiago: prevalencia de seis meses. Rev Psiquiatr 1994; 11: 194-202.

5. Vicente B, Rioseco P, Valdivia S, Kohn R, Torres S. Estudio chileno de prevalencia de patología psiquiátrica (DSM-III-R/CIDI) (ECPP). Rev Méd Chile 2002; 130: 527-36.

6. WHO. The World Health Report. Mental Health: New Understanding, New Hope. (c) World Health Organization 2001. Switzerland. 2001.

7. Ormel J, Von Korff M, Ustun TB, Pini S, Korten A, Oldenhinkel T. Common mental disorders and disability across cultures. Results from the WHO Collaborative study on psychological problems in general health care. JAMA 1994; 272: 1741-8. cia científica de que el tratamiento de la depresión en la atención primaria, puede ser más efectivo si la farmacoterapia que los médicos indican es monitorizada telefónicamente por personal entrenado y si los médicos generales son apoyados por personal especializado para la discusión de casos. Estos resultados parecen hoy muy importante dada la incorporación de la enfermedad depresiva como patología con garantías explícitas.

El equipo investigador se ha propuesto divulgar estos resultados y ofrecer apoyo para que los centros de atención primaria implementen monitorización telefónica de los pacientes que reciben tratamiento para la depresión a nivel primario.

8. Florenzano R, Acuña J, Fullerton C, Castro C. Estudio comparativo de frecuencia y características de los trastornos emocionales en pacientes que consultan en el nivel primario de atención en Santiago de Chile. Rev Méd Chile 1998; 126: 397-405.

9. Badamgarav E, Weingarten S, Henning J, Knight K, Hasselblad V, Gano A et al. Effectiveness of Disease Management Programs in depression: a systematic review. Am J Psychiatry 2003; 160: 2080-90.

10. National Institute of Mental Health. Breaking Ground, Breaking Through: The Strategic plan for Mood Disorders Research. National Institute of Mental Health. 2003.

11. Angst J. Clinical course of affective disorders. In: Helgason T, Daly RJ, eds. Depression Illness: Prediction of Course and Outcome. Berlin, Germany: Springer-Verlag; 1988; 1-47.

12. Haghighat R. Lifelong development of risk of recurrence in depressive disorders. J Affect Disord 1996; 41: 141-7.

13. Lin E, Vonkorff M, Russo J, Katon W, Simon G, Unutzer J ET AL. Can depression treatment in primary care reduce disability? A Stepped care approach. Arch Fam Med 2000; 9: 1052-8.

14. Ministerio de SAlud. Políticas y Plan Nacional de Salud Mental. Santiago. Chile. Ministerio de Salud. 1993.

15. Ministerio de Salud. Plan Nacional de Salud Mental y Psiquiatría. Santiago. Chile. Ministerio de Salud. 2000. 
16. Ministerio de Salud. «Guía Clínica para la Atención Primaria. La depresión, detección, diagnóstico y tratamiento». Santiago. Chile. Ministerio de Salud. 2001.

17. BAUER MS. A review of quantitative studies of adherence to mental health clinical practice guidelines. Harv Rev Psychiatry 2002; 10: 138-53.

18. Dietrich A, Oxman T, Williams J, Kroenke K, Schulberg $\mathrm{H}$, Bruce $\mathrm{M}$ et al. Going scale: reengineering systems for primary Care Treatment of Depression. Ann Fam Med 2004; 2: 301-4.

19. Dietrich A, Oxman T, Willaims J, Schulberg H, Bruce M, Lee P ET Al. Re-engineering systems for the treatment of depression in primary care: cluster randomized controlled trial. BMJ 2004; 329: 602 .

20. Simon G, Von Korff M, Rutter C, Wagner E. Randomized trial of monitoring, feedback, and management of care by telephone to improve treatment of depression in primary care. BMJ 2000; 320: 550-4.

21. Simon G, Revicki D, Vonkorff M. Telephone assessment of depression severity. J Psychiatr Res 1993; 27: 247-52.

22. Araya R, Wynn R, Lewis G. Comparison of two self administered psychiatric questionnaires (GHQ-12 and SRQ-20) in primary care in Chile. Soc Psychiatry Psychiatr Epidemiol 1992; 27: 168-73.

23. Johnstone A, Goldberg D. Psychiatric screening in general practice. A conttrolled trial. Lancet 1976; 1: 605-8.

24. Sheehan D, Lecrubier Y, Sheehan K, Amorim P, Janavs J, Weiller E et al. The Mini-International Neuropsychiatric Interview (M.I.N.I.): the development and validation of a structured diagnostic psychiatric interview for DSM-IV and ICD-10. J Clin Psychiatry 1998; 59 Suppl 20: 22-33; quiz 34-57.

25. Hamilton M. A rating scale for depression. J Neurol Neurosurg Psychiatry 1960; 23: 56-62.

26. Ware J, Sherbourne C. The MOS 36-item short-form health survey (SF-36). I. Conceptual framework and item selection. Med Care 1992; 30: 473-83.

27. Araya R, Rojas G, Fritsch R, Gaete J, Rojas M, Simon $G$ ET AL. Treating depression in primary care in low-income women in Santiago, Chile: a randomized controlled trial. Lancet 2003; 361: 995-1000.

28. STATA 8.0, College St, Texas.

29. Gilbody S, Whitty P, Grimshaw J, Thomas R. Educational and organizational interventions to improve the management of depression in primary care: a systematic Review. JAMA 2003; 289: 3145-51.

30. Hunkeler E, Meresman J, Hargreaves W, Fireman B, Berman W, Kirsch A et al. Efficacy of nurse telehealth care and peer support in augmenting treatment of depression in primary care. Arch Fam Med 2000; 9: 700-8.

31. Curry S, Mcbride C, Grothaus L, Louie D, Wagner E. A randomized trial of self-help materials, personalized feedback, and telephone counselling with nonvolunteer smokers. J Consult Clin Psychol 1995; 63: 1005-14.

32. Litzelman D, Dittus R, Miller M, Tierney W. Requiring physicians to respond to computerized reminders, improves their compliance with preventive care protocols. J Gen Intern Med 1993; 8: 311-7.

33. McDonald C, Hui S, Smith D, Tierney W, Cohen S, WeINBERger M ET AL. Reminders to physicians from an introspective computer medical record. A twoyears randomized trial. Ann Intern Med 1984; 100: $130-8$.

34. Weingarten S, Henning J, Badamgarav E, Knight K, Hasselblad V, Gano A et al. Interventions used in disease management programmes for patients with chronic illness-which ones work? Metaanalysis of published reports. BMJ 2002; 325 : 925-8.

35. Alvarado R, Vega J, Sanhueza G, Muñoz MG. Evaluación del programa para la detección, diagnóstico y tratamiento integral de la depresión en atención primaria, en Chile. Rev Panam Salud Pública 2005; 18(4/5): 278-86. 